\title{
Optimization Approach of the Vehicle Routing Problem with Packing Constraints Using Genetic Algorithm Method
}

\author{
Nurlita Gamayanti, Yusuf Bilfaqih, Rusdhianto EAK, M. Yasya BU \\ Department of Electrical Engineering \\ Sepuluh Nopember Institute of Technology \\ Surabaya, Indonesia \\ $\underline{\text { lita@ee.its.ac.id, lita@ee.its.ac.id }}$
}

\begin{abstract}
Vehicle Routing Problem is an issue in item delivery from depot to its customers using several vehicles which have limited capacity with a purpose to minimize transportation cost. The packing constraints exist because the vehicles which are usually used in item delivery have rectangular-box shaped container. Also, the items are commonly in shape of rectangular-box. Therefore, packing or loading method is needed so that containers could load all of the items without causing damage and could ease unloading process. The purpose of this final project is to develop a model and algorithm using metaheuristics method, especially genetics algorithm in order to minimize total delivery distance. A hybrid genetics algorithm and bottom-left fill algorithm also take place to solve the packing process. This algorithm delivered average solution $0.08 \%$ worse than ant colony optimization, but had $2.93 \%$ better solution than tabu search.
\end{abstract}

Keywords - Routing, Optimization, Genetics Algorithm, Packing, Bottom-Left Fill

\section{INTRODUCTION}

\section{A. Background}

Transportation is one of the important components in item delivery or logistics management. The increase of efficiency must be performed because the cost of delivery is proportional to the distance or travel time of the vehicle. In addition, the increase of vehicle utility is also required for the proper delivery process. In this case, it is necessary to find the best paths and routes which are able to minimize the time and distance as well as to increase the utility of each vehicle. The problem which aims to make the best route on a group of delivery vehicles is called Vehicle Routing Problem (VRP).

In general, VRP is modeled using graph theory by designing depot or customer nodes and arc on the connecting path between nodes. Then, it creates a flow from the depot node to a group of scattered customer nodes at the cheapest cost. The flow is constructed so that the service given to each customer node is in accordance with the demand and the total carrying quantity must not exceed the vehicle capacity. In the case study, it is assumed that the vehicle is a box-truck with a rectangular-box shaped containing pallets to organize items. The items shipped must be arranged so that they fit on the vehicle, do not damage each other and ease the unloading process (items from the early visited customers are located closer to the door), such constraints are called Packing Constraints (PC).

Genetics algorithm is a method which is able to generate permutations continuously to obtain better solution then the best results are taken to obtain the most optimal result (objective function).

\section{MODEL AND DESIGN}

\section{A. Vehicle Routing Problem with Packing Constraints and} Model Development

In scheduling of Vehicle Routing Problem with Packing Constraints, a model which is able to describe the problem and simplify the use of algorithms is created. The concept of this model aims to determine the tour of each delivery vehicle from the depot to the customer nodes then back again to the depot along with the determination of how to organize the items to be delivered. The objective of determining the tour of each vehicle is to minimize the distance traveled. Input control and action variables used are the determination of each tour route and the packing of items into the vehicle. Alternative action which is allowed is increasing the number of vehicles.

The Vehicle Routing Problem with Packing Constraints model was originally derived from Vehicle Routing Problem and Packing Constraint based on 3-Dimensional Bin Packing Problem and then developed.

Vehicle Routing Problem is a continuation of the Traveling Salesman Problem which is a problem of finding the shortest distance to visit all nodes exactly once on a graph and back to the starting node using one vehicle. Then the model develops to Scheduling issue which has more than one vehicle required to meet the demands of each node. Each vehicle from the depot node visiting several nodes then returning to the depot node is called tour. [1]

This problem can be expressed in the form of directed graph which containing a set of nodes and $\operatorname{arc}(N, A)$. Where $N=\{0,1,2, \ldots, n\}$ is a set of nodes representing the customers, the city, the destination or place to which it is demanded. While $A$ is the arc set which represents the distance or travel time of the two nodes. Then each vehicle 
forms a network in the form of a cycle starting at the depot and back to the depot. [2]

Scheduling in the VRP is classified in problem with computational time complexity of NP (Nondeterministic Polynomial time). Therefore the algorithm with polynomial time complexity used to obtain the most optimal solution has not been found. Some heuristic and metaheuristic algorithms are presented, but they have not been able to produce optimal values, only close to optimal results. [2]

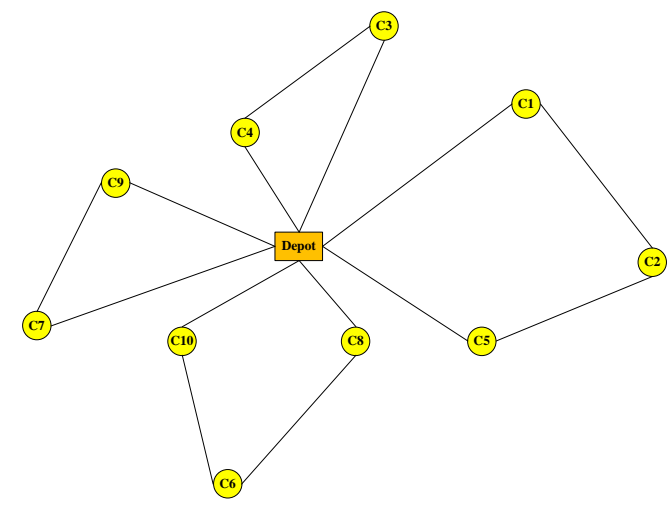

Figure 1. Graph Representation of VRP

There are many kinds of Vehicle Routing Problems. VRP which often being used is VRP using additional capacity constraints. The mathematical models used are as follow [2]

$$
\begin{array}{lc}
\text { Minimize } \quad \sum_{i} \sum_{j} \sum_{k} c_{i j} \psi_{i j k} \\
\begin{array}{l}
\text { Subject to } \\
\sum_{j \in V_{0}} \sum_{k}^{\prime} \psi_{i j k}=1
\end{array} \\
\sum_{i \in V}^{\prime} \psi_{i 0 k} \leq 1 & \forall i \in V \\
\sum_{i \in V_{0}} \psi_{i h k}-\sum_{j \in V_{0}}^{\prime} \psi_{h j k}=0 & \forall h \in V_{0}, k \\
\sum_{j \in V} \sum_{k} \psi_{0 j k} \geq 1 & \\
u_{i}-u_{j}+n m \sum_{k} \psi_{i j k}=n m+1 \\
\forall i, j \in V \\
\sum_{i \in V} \sum_{j \in V_{0}} m_{i} \psi_{i j k} \leq Q_{k}
\end{array}
$$

The model's Objective Function, (1), is to minimize the delivery distance where $\psi_{i j k}$ represents whether vehicle $k$ =moves from node $i$ to $j$ and $c_{i j}$ represents the distance of movement from $i$ to $j$. Equation (2) states that each customer node is serviced by exactly one vehicle. $V$ is the set of all customer nodes and $V_{0}$ is the set of all customers and depot nodes. Inequality (3) states that every vehicle does the tour at most once. Equation (4) states that every vehicle from a node can move to another node only. Inequality (5) states there is at least one vehicle operating. Equation (6) is used to remove subtours that do not pass through the depot, while $u_{i}$ denotes the order of visits and $\mathrm{nm}$ is the number of customer nodes. Inequality (7) states that the weight carried by the vehicle to serve the customer nodes must not exceed its capacity. While, $m_{i}$ denotes the demand's weight of node $i$ and $Q_{k}$ is the capacity of vehicle $k$.

Once VRP model is obtained then 3D-BPP model is applied as constraint. Bin Packing Problem is a problem of organizing some objects into a limited container with a specific purpose, for example to fit all objects into a container according to the existing constraints or maximize incoming items according to priority of items and the contraints which exist (Knapsack Problem). In the calculation process this problem is included in the classification of NP-Hard problems. Therefore various heuristic algorithms are presented such as first-fit which allows items to be loaded in certain order but it does not guarantee the results achieved is optimal.

The Three-Dimensional Bin Packing Problem is a part of BPP where a rectangular-box shaped object can be rotated 90 degrees horizontally with a rectangular-box shaped container and the sides of all objects are parallel to the sides of the container. This problem is similar to 2D-BPP and some of the algorithms used to solve 2D-BPP can also be used to solve 3D-BPP. However, in real condition each item is affected by gravity so there needs to be a minimum support area, a support capacity, and objects' balance.

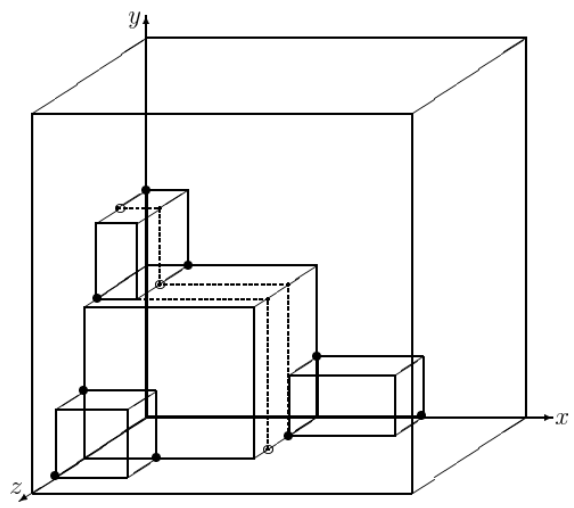

Figure 2. Three-Dimensional Bin Packing Problem [3]

This problem can be defined as the problem of packing and arrangement of items defined in set $\{1,2,3, \ldots, n\}$ where each item, has the width of $w_{i}$, length of $l_{i}$, and height of $h_{i}$ to a container which has width $W$, length $L$ and height $H$. Furthermore, the variables that can be determined from this packing process are the coordinates of the items. Suppose that the $x, y, z$ axes are parallel to the length, width, and height of the container with an origin located on the bottomleft-rear corner of the container. While, the coordinate variable of item $i$ is $\left(x_{i}, y_{i}, z_{i}\right)$. In general, the objective function of the problem is simply to find a possible solution or feasible solution. However, there is also a model which objective is to maximize the occupational volume of the items. The following equations represent a mixed integer linear programming model for 3D-BPP on vehicle with length $L$, width $W$ and height $H$, while the size of each item $\alpha$ is $p_{\alpha}$ in length, $q_{\alpha}$ in width and $r_{\alpha}$ in height.

$$
\begin{aligned}
& x_{\alpha}+p_{\alpha} l x_{\alpha}+q_{\alpha} w x_{\alpha}+r_{\alpha} h x_{\alpha} \leq x_{\beta}+\left(1-a_{\alpha \beta}\right) M \\
& \forall \alpha, \beta
\end{aligned}
$$




$$
\begin{aligned}
& x_{\beta}+p_{\beta} l x_{\beta}+q_{\beta} w x_{\beta}+r_{\beta} h x_{\beta} \leq x_{\alpha}+\left(1-b_{\alpha \beta}\right) M \\
& \forall \alpha, \beta \\
& y_{\alpha}+p_{\alpha} l y_{\alpha}+q_{\alpha} w y_{\alpha}+r_{\alpha} h y_{\alpha} \leq y_{\beta}+\left(1-c_{\alpha \beta}\right) M \\
& \forall \alpha, \beta \\
& y_{\beta}+p_{\beta} l y_{\beta}+q_{\beta} w y_{\beta}+r_{\beta} h y_{\beta} \leq y_{\alpha}+\left(1-d_{\alpha \beta}\right) M \\
& \forall \alpha, \beta \\
& z_{\alpha}+p_{\alpha} l z_{\alpha}+q_{\alpha} w z_{\alpha}+r_{\alpha} h z_{\alpha} \leq z_{\beta}+\left(1-e_{\alpha \beta}\right) M \\
& \forall \alpha, \beta \\
& z_{\beta}+p_{\beta} l z_{\beta}+q_{\beta} w z_{\beta}+r_{\beta} h z_{\beta} \leq z_{\alpha}+\left(1-f_{\alpha \beta}\right) M \\
& \forall \alpha, \beta \\
& a_{\alpha \beta}+b_{\alpha \beta}+c_{\alpha \beta}+d_{\alpha \beta}+e_{\alpha \beta}+f_{\alpha \beta} \geq 1 \quad \forall \alpha, \beta \\
& x_{\alpha}+p_{\alpha} l x_{\alpha}+q_{\alpha} w x_{\alpha}+r_{\alpha} h x_{\alpha} \leq L \quad \forall \alpha, \beta \\
& y_{\alpha}+p_{\alpha} l y_{\alpha}+q_{\alpha} w y_{\alpha}+r_{\alpha} h y_{\alpha} \leq W \quad \forall \alpha, \beta \\
& z_{\alpha}+p_{\alpha} l z_{\alpha}+q_{\alpha} w z_{\alpha}+r_{\alpha} h z_{\alpha} \leq H \quad \forall \alpha, \beta \\
& l x_{\alpha}+l y_{\alpha}+l z_{\alpha}=1 \quad \forall a \\
& w x_{\alpha}+w y_{\alpha}+w z_{\alpha}=1 \quad \forall a \\
& h x_{\alpha}+h y_{\alpha}+h z_{\alpha}=1 \quad \forall a \\
& l x_{\alpha}+w x_{\alpha}+h x_{\alpha}=1 \quad \forall a \\
& l y_{\alpha}+w y_{\alpha}+h y_{\alpha}=1 \quad \forall a \\
& l z_{\alpha}+w z_{\alpha}+h z_{\alpha}=1 \quad \forall a
\end{aligned}
$$

The variables $x_{\alpha}, y_{\alpha}$ and $z_{\alpha}$ are coordinates of the items $\alpha$ and $l x_{\alpha}, l y_{\alpha}, l z_{\alpha}, w x_{\alpha}, w y_{\alpha}, w z_{\alpha}, h x_{\alpha}, h y_{\alpha}, h z_{\alpha}$ are binary variables of the items position decision. Equations (2.8), (2.9), (2.10), (2.11), (2.12), (2.13) and (2.14) explain the relationship between two items so that no overlapping happen between items with $a_{\alpha \beta}, b_{\alpha \beta}, c_{\alpha \beta}, d_{\alpha \beta}, e_{\alpha \beta}, f_{\alpha \beta}$, representing whether $\alpha$ is consecutively on the left, right, in front, behind, below, or above item $\beta$. Equations (2.15), (2.16) and (2.17) indicate that the items are loaded inside the container. Equations (2.18), (2.19), (2.20), (2.21), (2.22), (2.23) indicate the position of the items being laid. After assigning 3D-BPP model as Packing Constraints, development is continued to obtain a model which is close to reality.

The development applied to the existing model compared to the model used in the literature study is the addition of constraints on the scheduling and the process of item arrangement in the form of

1. All vehicles are identical, meaning every vehicle has the same cost per unit distance, container size, and weight capacity

2. Last-in First-Out (LIFO) Constraint which aims to ease officers' duty in loading process. This constraint is stated when item $\alpha$ needs to be delivered earlier than item $\beta$, then item $\alpha$ should not be under or behind item $\beta$.

3. Minimum support area. This constraint aims to ensure that the items supported are physically balance. This relationship is expressed in the ratio of the surface area of the supported sector of supported items and the total surface area of the supported items to be greater than the constant, $F<1$.

4. Fragility, the existence of fragile items which is not able in supporting other items.

5. Rotation, only horizontal rotation is allowed.

\section{B. Genetic Algorithm for VRP}

In this step, genetics algorithm is developed to solve the Vehicle Routing Problem with Packing Constraints. This development comes from the genetics algorithms adapted to the VRP problem [G. Vaira and O. Kurasova, 2013] which then will be used to solve Packing Constraints. The input of this algorithm is the data which was used in the previous research [G. Fuellerrer. K.F. et al, 2010] which is the distance between the depot node and the customer node. This algorithm is required to get the best fitness solution that meets the primary constraints of weight capacity and occupational volume, and secondary constraints in the form of item packing. Secondary constraint testing is performed with a combination of genetics algorithms and completeBLF. The following algorithm of VRP is used.

1. Initialize data and parameter values.

Parameter value is determined by reference and trial and error, then selected the best parameter value

2. Generate the best initial individuals set where all the best individuals must meet the primary and secondary constraints

3. Generate initial individuals. All individuals in the population must meet the primary constrain. There are two kinds of initial generation which are cluster generation and random generation

4. Set the fitness value and secondary constraint test, if there are individuals which meet the secondary constrain with better fitness value. Put individuals into the best individuals set and remove one individual which have the worst fitness from the best individuals set

5. Apply crossover, using Improved Route Based Crossover (IRBX) and Improved Sequence Based Crossover (ISBX) by ensuring the fulfillment of primary constrain. If the offspring which is produced in crossover, has worse fitness or does not meet the primary constraint then keep the parents in the population, otherwise put the individual offspring into the population .

ISBX and IRBX are the development of Sequence Based Crossover (SBX) and Route Based Crossover (RBX) algorithms to produce better solutions by replacing the random factor on both SBX and RBX with roulette wheel factor.

6. Do mutation. There are 2 types of mutation, those are the generation of new individuals and 2opt algorithm. New individual generation is used so that results are not stuck on the local optimal. While 2opt algorithm is used to 


\section{RESULTS AND ANALYSIS}

The data which is used is presented as secondary data from the previous research [G. Fuellerrer. K.F. et al, 2010]. These data consisted of 15 customers, 26 items, and 5 vehicles. While results obtained are

1. probability of cluster generation is 0.8 while random generation is 0.2

2. probability of ISBX is 0.4 while IRBX is 0.6

3. probability of new individual generation is 0.1 while 2opt Algorithm is 0.8

Then, the result obtained is the shortest distance (in unit) which is presented in Table 1.

TABLE 1. SHORTEST PATH (UNIT) PEFORMANCE COMPARISSON OF GENETIC ALGORITHM (GA) WITH TABU SEARCH [M. GENDREAU, 2006] AND ANT COLONY OPTIMIZATION [G. FUELLERER, K ET AL, 2010]

\begin{tabular}{|l|c|c|c|c|c|}
\hline \multicolumn{1}{|c|}{} & \multicolumn{5}{|c|}{ Instance } \\
\cline { 2 - 6 } & 2 & 4 & 6 & 16 & 17 \\
\hline $\begin{array}{l}\text { GA } \\
\text { Mean }\end{array}$ & 334.96 & 440.68 & 501.42 & 698.92 & 872.68 \\
\hline $\begin{array}{l}\text { ACO } \\
\text { Mean }\end{array}$ & 334.96 & 440.68 & 501.47 & 698.92 & 870.33 \\
\hline $\begin{array}{l}\text { TS } \\
\text { Mean }\end{array}$ & 350.58 & 448.48 & 504.46 & 707.85 & 920.87 \\
\hline
\end{tabular}

It can be seen in Table 1 that the average value of genetic algorithm results lag $0.08 \%$ compared to the result of ACO but $2.93 \%$ better than Tabu Search.

\section{CONCLUSION}

After performing analysis of the test result, the conslusion obtained from comparison and implementation in real condition are as follow

1. The results of the Genetic Algorithm are strongly influenced by parameters used such as generation, crossover and mutation.

2. The genetic algorithm has a solution which is $0.08 \%$ worse than ACO but $2.93 \%$ better than Tabu Search

\section{REFFERENCE}

[1] R. K. Ahuja, T. L. Magnanti and J. B. Orlin, Network Flows, Pearson, 1993.

[2] G. Laporte, "The Vehicle Routing Problem : An overview of exact and approximate algorithms," European Journal of Operational Research, pp. 345-358, 1992.

[3] S. Martello, D. Pisinger and D. Vigo, "The Three-Dimensional Bin Packing Problem," Operations Research, pp. 256-267, 2000.

[4] G. Vaira and O. Kurasova, "Genetic Algorithms and VRP: the Behaviour of a Crossover Operator," Baltic Journal of Modern Computing, 2013.

[5] G. Fuellerer, K. F. Doerner, R. F. Hartl and M. Iori, "Metaheuristic for vehicle routing problems with three-dimensional loading constraints," European Journal of Operational Research, pp. 751759,2010

[6] M. Hifi, I. Kacem, S. Negre and L. Wu, "A Linear Programming Approach for the Three-Dimensional Bin Packing Problem," Electronic Notes in Discrete Mathematics, pp. 993-1000, 2010.

[7] I. Prasetyaningrum, "Penyelesaian Kombinasi Vehicle Routing Problem dan Container Loading Problem Menggunakan Algoritma Genetika," Surabaya, 2007.

[8] D. Liu and H. Teng, "An Improved BL-algorithm for genetic algorithm of the orthogonal packing of rectangles," European Journal of Operational Research, pp. 413-420, 1999.

[9] S. Imahori, M. Yagiura and H. Nagamochi, "Practical Algorithms for Two-dimensional Packing," Mathematical Engineering Technical Reports, Tokyo, 2006.

[10] B. S. Baker, E. Coffman, JR and R. L. Rivest, "Orthogonal Packings in Two Dimensions," SIAM Journal on Computing, pp. 846-855, 1980.

[11] M. Gendreau, M. Iori, G. Laporte and S. Martello, "A Tabu Search Algorithm for a Routing and Container Loading Problem," Transportation Science, pp. 342-350, 2006

[12] M. Gendreau, M. Iori, G. Laporte and S. Martello, "A Tabu Search heuristic for the vehicle routing problem with two dimensional loading constraints," Networks, p. Forthcoming, 2006.

[13] M. Iori and S. Martello, "Routing problem with loading constraint," TOP, 2010 .

[14] B. Santosa and P. Willy, Metoda Metaheuristik Konsep dan Implementasi, Surabaya: Guna Widya, 2011.

[15] A. Turkay and E. Emel, "Vehicle Routing Problem with Packing Contraints," in 5th Euro/Informs Joint International Meeting, Istanbul, 2003. 